\title{
A CULTURA EM TORNO DA SEGURANÇA DO PACIENTE NA ATENÇÃO PRIMÁRIA À SAÚDE: DISTINÇÕES ENTRE CATEGORIAS PROFISSIONAIS
}

\author{
THE CULTURE REGARDING THE SAFETY OF THE PATIENT IN PRIMARY HEALTH \\ CARE: DISTINCTIONS AMONG PROFESSIONAL CATEGORIES
}

\begin{abstract}
Lilian Louzada Macedo' (D) [0000-0003-3166-4283], Ana Maria Rigo Silva² (DD [0000-0002-9527-4914], João Felipe Marques da Silva² (DD [0000-0001-7198-8528], Maria do Carmo Fernandez Lourenço Haddad $^{3}$ (iD) [0000-0001-7564-8563], Edmarlon Girotto ${ }^{2}$ (iD) [0000-0001-9345-3348]

\author{
1 Secretaria de Saúde, município de Governador Celso Ramos, Santa Catarina, Brasil. \\ ${ }^{2}$ Universidade Estadual de Londrina, Centro de Ciências da Saúde, Programa de Pós-Graduação em \\ Saúde Coletiva, Londrina, Paraná, Brasil. <edmarlon78@gmail.com> \\ ${ }^{3}$ Universidade Estadual de Londrina, Centro de Ciências da Saúde, Programa de Pós-Graduação em \\ Enfermagem, Londrina, Paraná, Brasil.
}

Resumo O estudo que originou este artigo teve como objetivo analisar a cultura de segurança do paciente na atenção primária em saúde de um município de grande porte do estado do Paraná, segundo a categoria profissional. Foi desenvolvido um estudo transversal com trabalhadores da atenção primária, com coleta de dados realizada em 2017, por meio da aplicação de um instrumento autorrespondido denominado Pesquisa sobre Cultura de Segurança do Paciente para Atenção Primária (Medical Office Survey on Patient Safety Culture). Os resultados indicaram que o processo de trabalho no serviço de atuação e apoio dos gestores foram as dimensões da cultura de segurança com avaliação mais fragilizada. De modo geral, houve divergências na percepção da cultura de segurança pelos profissionais de saúde da atenção primária, denotando a importância do planejamento conjunto de estratégias de assistência à saúde. Os técnicos de enfermagem apresentaram maior prevalência de avaliação fragilizada da cultura de segurança do paciente. De forma contrária, os enfermeiros mostraram menor prevalência de avaliação fragilizada sobre a cultura de segurança.

Palavras-chave segurança do paciente; atenção primária à saúde; trabalhadores da saúde.
Abstract The study that originated the present article had the goal of analyzing the culture of patient safety in primary health care in a large municipality in the state of Paraná, Brazil, according to the professional category. We conducted a cross-sectional study with primary health care workers, and the data was collected in 2017 through the application of a self-reported instrument called Survey on Patient Safety Culture for Primary Health Care (Pesquisa sobre Cultura de Segurança do Paciente para Atenção Primária, in Portuguese). The results indicated that the work process in each service where they work and the support from the managers were the dimensions of the safety culture that had the weakest evaluations. In general, there were differences of opinions regarding the perception of the safety culture on the part of the primary health care professionals, indicating the importance of the joint planning of health care strategies. The nurse technicians revealed a higher prevalence of weak evaluations of patient safety culture. On the other hand, the nurses revealed a lower prevalence of weak evaluations regarding safety culture.

Keywords patient safety; primary health care; health workers. 


\section{Introdução}

A segurança do paciente tem como objetivo reduzir os riscos associados à assistênciaàsaúde, provenientes detecnologiaseprodutos, relaçõeshumanasno serviço e falhas na comunicação com o paciente (Luiz e Cohn, 2006). No ambientehospitalar, as ações promotoras de segurança do pacientee preventivas deeventoscausadoresdedanosàsaúdevêmsendodelimitadasereconhecidas pela gestão e pelos demais profissionais. No entanto, tal temática não tem a mesma visibilidade na atenção primária (Marchon, 2014).

A atenção primária em saúde é responsável por resolver até $80 \%$ dos problemas de saúde da população (Brasil, 2015). Dessa forma, entende-se que há uma diversidade de situações de saúde a serem solucionadas nesse nível de atenção, que exigem do profissional habilidade, conhecimento técnico e atitudes de empatia, e envolvem a escuta qualificada e a personalização do atendimento, entre outras. No entanto, a atuação do profissional na assistência a essas situações de saúde é passível de falhas, sejam elas técnicas, sejam provenientes da ausência de atitudes de empatia (Hubert, 2018).

Estudo realizado em 2012 na Suíça, o qual avaliou médicos e enfermeiros sobre a frequência e gravidade de incidentes relacionados à segurança do paciente na atenção primária à saúde (APS), revelou a média de 92 incidentes por profissional durante os 12 meses anteriores à pesquisa. Identificou-se ainda que cerca de metade dos incidentes trouxe danos graves à saúde ou levaram à morte, e que em sua maioria esses danos estavam relacionados a erros diagnósticos (Gehring et al., 2012).

De acordo com Marchon, Mendes Júnior e Pavão (2015), os erros administrativos, de comunicação e no tratamento foram relatados por profissionais das secretarias municipais de uma baixada litorânea do Rio de Janeiro como os mais frequentes na APS. Logo, entende-se que é real a ocorrência e gravidade de erros na APS, e que esses, em sua maioria, são falhas que se encontram sob a governabilidade dos profissionais desse nível de atenção para serem identificadas e evitadas.

Dentre os trabalhadores que compõem as equipes de saúde da família, encontram-se agentes comunitários, técnicos e/ou auxiliares de enfermagem, enfermeiros, médicos e equipe odontológica (Brasil, 2017). Esses trabalhadores têm responsabilidades e atuações diferentes, entre elas assistir o paciente de acordo com suas formações técnicas e pessoais. Portanto, cada um constrói percepções diversificadas quanto à segurança do paciente. Nesse contexto, o objetivo do estudo que deu origem a este artigo foi analisar a cultura de segurança do paciente na APS de um município de grande porte segundo a categoria profissional. Essa compreensão poderá ser subsídio para o desenvolvimento de ações mais específicas e significativas para os trabalhadores da equipe da APS, 
proporcionando assim a construção de uma cultura de segurança do paciente mais positiva (Paese e Dal Sasso, 2013).

\section{Métodos}

Trata-se de um estudo de delineamento transversal, abrangendo trabalhadores atuantes na APS de Londrina, Paraná. O município apresentava, segundo estimativa do Instituto Brasileiro de Geografia e Estatística (IBGE), 558.439 habitantes em 2017 (IBGE, 2018). Ademais, contava com 54 unidades de saúde da família (USFs), 42 localizadas na área urbana do município no período de pesquisa.

A população de estudo foi composta por todos os trabalhadores atuantes nas unidades de saúde da família da área urbana de Londrina, excluindo-se unidades que oferecem assistência por 16 horas diárias (duas USFs). Estas foram excluídas, pois apresentam fragilidade no vínculo profissional-usuário e profissionalprofissional, considerando a atuação pontual de plantonistas e em razão de se distinguirem das demais USFs devido ao fluxo e à quantidade de atividades.

Das unidades selecionadas, foram excluídos profissionais do Núcleo de Apoio à Saúde da Família (Nasf), profissionais residentes, internos e estagiários das instituições de ensino superior ou ensino técnico, pois entende-se que estes têm atuação parcial ou pouco sólida na unidade e equipe de saúde, e inserção sólida no processo de trabalho da unidade. Além destes, excluíram-se os trabaIhadores de serviços gerais, devido ao menor vínculo que demonstraram possuir com a equipe de saúde, pois são, em sua grande parte, terceirizados, e ao menor vínculo com os pacientes quando comparados com as demais categorias. Todas essas exclusões visaram garantir que os respondentes conhecessem o suficiente a unidade e o processo de trabalho em que estavam inseridos para que fornecessem respostas de qualidade (Sorra, Franklyn e Streagle, 2008).

Para a coleta de dados, foi utilizado o instrumento Pesquisa sobre Cultura de Segurança do Paciente para Atenção Primária (MOSPSC - Medical Office Survey on Patient Safety Culture), formulado em 2007 (AHRQ, 2016), traduzido, adaptado e validado para a atenção primária no Brasil em 2015 (Timm e Rodrigues, 2016; Timm, 2015). O instrumento, autopreenchido, contou com questões adicionais elaboradas para a obtenção de dados sociodemográficos.

$\mathrm{O}$ instrumento MOSPSC dispõe de oito seções, de $\mathrm{A}$ a $\mathrm{H}$, que abordam a percepção e a avaliação do profissional quanto à segurança do paciente. Para este estudo, foram utilizadas as seções $C$ a $G$, as quais tratam das seguintes dimensões: 'trabalhando neste serviço de saúde' (seção $C$ ); 'comunicação e acompanhamento' (seção D); 'apoio de gestores/administradores/líderes' (seção E); 'seu serviço de saúde' (seção F); e 'avaliação global' (seção G). Os participantes da pesquisa foram informados, antes de responderem ao questionário, que a expressão gestores/administradores/líderes deveria ser interpretada como profis- 
sionais coordenadores das USFs. Além disso, esses profissionais (coordenadores das USFs) não responderam à seção $E$.

Previamente à coleta de dados, foi realizado um estudo-piloto em duas USFs de um município de médio porte localizado próximo a Londrina. Por meio do estudo-piloto, identificaram-se possíveis obstáculos para a aplicação do questionário, dúvidas quanto ao processo de coleta de dados em sua completude, mediante a reprodução de todos os métodos e estratégias propostas para o estudo. Tal estudo também foi útil para teste definitivo dos instrumentos, buscando, como apresenta a literatura, afirmar a viabilidade deles (Klein e Bloch, 2008).

A coleta de dados foi realizada nas dependências das USFs. Para isso, os pesquisadores contataram inicialmente as coordenações das unidades para acordar detalhes da pesquisa, por e-mail ou telefone. Devido ao não sucesso na tentativa de contato com algumas coordenadoras, optou-se por participar de uma reunião técnica municipal com todas elas para informá-las sobre o objetivo da pesquisa e o processo de coleta de dados. Após essa reunião, decidiu-se por realizar a coleta de dados sem agendamento prévio de dia e horário. Quando não foi possível a aplicação dos questionários no mesmo dia a todos os trabalhadores, as pesquisadoras retornaram em dias posteriores para a coleta. A coleta de dados iniciou-se em abril de 2017 e finalizou-se em junho de 2017. Foi solicitado o número de funcionários às coordenadoras no dia da coleta, o que proporcionou a identificação daqueles ausentes no dia ou período entre a entrega dos questionários e seu recolhimento. Realizaram-se duas visitas posteriores para a busca desses trabalhadores.

As variáveis de caracterização foram: faixa etária (até 25 anos; de 26 a 50 anos; de 51 a 66 anos), sexo (feminino; masculino), tempo de trabalho na USF (menos de 3 anos; de 3 a menos de 6 anos; de 6 a menos de 11 anos; há 11 anos ou mais), curso/capacitação em segurança do paciente (sim; não), outro vínculo empregatício (sim; não) e carga horária semanal no serviço (24 horas ou menos; de 25 a 32 horas; de 33 a 40 horas; 41 horas ou mais). A variável independente neste estudo foi a categoria profissional, estratificada em: enfermeiro (incluindo enfermeiro e enfermeiro coordenador), técnico de enfermagem, agente comunitário de saúde (ACS), médico, equipe de odontologia (incluindo cirurgião-dentista e auxiliar de odontologia) e equipe administrativa.

As variáveis dependentes do estudo foram as seções $C$ a $G$ do instrumento MOSPSC. Para as seções $C$ a F, foram analisados os percentuais de respostas positivas para cada dimensão do instrumento, utilizando-se o seguinte cálculo (Sorra, Franklyn e Streagle, 2008): [número de respostas positivas aos itens da dimensão / número total de respostas válidas aos itens da dimensão (positivas, neutras e negativas, excluindo-se os dados ausentes)] x 100. Esta análise foi aplicada por profissional, identificando o percentual de respostas positivas por indivíduo entrevistado. 
Consideraram-se respostas positivas aquelas em que foram assinaladas 'concordo totalmente/parcialmente' ou 'quase sempre/sempre' para as sentenças formuladas de forma positiva, ou 'discordo totalmente/parcialmente' ou 'nunca/raramente' para as perguntas formuladas negativamente. As respostas neutras referem-se àquelas em que foram assinaladas 'nem discordo nem concordo' ou 'às vezes' para qualquer pergunta. As respostas negativas referem-se àquelas em que foram assinaladas as opções 'discordo totalmente/parcialmente' ou 'nunca/raramente' para as perguntas formuladas de forma positiva, ou 'concordo totalmente/parcialmente' ou 'quase sempre/sempre' nas sentenças formuladas negativamente (Sorra, Franklyn e Streagle, 2008).

Ainda compondo a percepção sobre segurança do paciente (seção G), os profissionais realizaram uma avaliação geral da qualidade do cuidado, em que se atribuíram percepções de ruim a excelente para os serviços de saúde. Foram consideradas 'ruim' e 'razoável' como percepção negativa, 'bom' como percepção neutra, e'muito bom' e 'excelente' como positivas, sendo calculado o percentual de respostas positivas para essa dimensão da mesma forma que calculado para as seções mencionadas anteriormente.

Com base no cálculo dos percentuais de respostas positivas obtidos para as dimensões da cultura de segurança, consideraram-se fortalecidas as dimensões que alcançaram $75 \%$ ou mais de respostas positivas. Quando o percentual se apresentasse maior que $50 \%$ e menor que $75 \%$, ela foi considerada com potencial de melhoria; por fim, caso o percentual de respostas positivas fosse de $50 \%$ ou menos, considerou-se fragilizada.

As informações obtidas por meio dos instrumentos de coleta de dados foram duplamente digitadas por distintos pesquisadores em planilha criada no programa Microsoft Excel 2013. Ambos os arquivos foram comparados no programa Epi Info, versão 3.5.4 para Windows, e os dados discrepantes corrigidos após consulta aos instrumentos de coleta. O processamento e a análise dos dados foram realizados utilizando-se o programa Statistical Package for the Social Sciences (SPSS), versão 19.0, para Windows.

Para as análises de associação, utilizou-se a regressão de Poisson com cálculo da razão de prevalência (RP) e intervalo de 95\% de confiança (IC95\%), empregando-se como nível de significância $p<0,05$. Assim, as variáveis de cultura de segurança foram dicotomizadas em 'fortalecidas' e 'fragilizadas/ potencial de melhoria', sendo esta última denominada neste estudo de cultura de segurança 'fragilizada'. Usou-se como variável independente a categoria profissional, sendo que cada categoria foi comparada, individualmente, com as demais. Foram utilizadas como variáveis de ajuste: faixa etária, sexo e tempo de trabalho no serviço. Também se avaliou a consistência interna do instrumento utilizado (MOSPSC). Para isso, foi calculado o coeficiente alfa de Cronbach (Cronbach, 1951). 
O estudo foi aprovado pelo Comitê de Ética em Pesquisa da Universidade Estadual de Londrina (CEP-UEL) - Certificado de Apresentação para Apreciação Ética (CAAE) n. 62763916.4.0000.5231. Cada participante foi devidamente orientado quanto aos objetivos da pesquisa, tendo lido e assinado o termo de consentimento livre e esclarecido (TCLE).

\section{Resultados}

O município de Londrina contabiliza 1.231 funcionários nas USFs da zona urbana que têm horário de funcionamento de até 12 horas diárias. Destes, 65 pertencem ao Nasf e 23 ocupam o cargo de serviços gerais. Além disso, 12 não estavam listados no quadro de funcionários das unidades, tornando-se elegíveis para esta pesquisa 1.131 profissionais de saúde atuantes na APS. Dentre estes ( $n=1.131)$, foram identificadas $403(35,6 \%)$ recusas, 134 (11,8\%) profissionais não encontrados, 44 (3,8\%) de férias ou em afastamento e 37 (3,3\%) que não informaram a categoria profissional ou informaram 'outra função', sem especificá-la - totalizando, para este estudo, uma amostra de 513 profissionais (45,4\% de taxa de resposta).

Os profissionais que mais aderiram à pesquisa foram os enfermeiros e agentes comunitários de saúde (60,8\% e 58,9\% de taxa de resposta, respectivamente). De forma contrária, os médicos apresentaram taxa de resposta de apenas $23,5 \%$, conforme pode ser verificado na Tabela 1.

A maioria dos participantes era do sexo feminino $(83,2 \%)$, com idades entre 26 e 50 anos (73,3\%); 78,2\% não relataram curso/capacitação em segurança do paciente e $82,9 \%$ não referiram outro vínculo empregatício. A idade média foi de 42 anos (desvio padrão: 9,5 anos). Também se deve destacar que cerca de $70 \%$ trabalhavam há três anos ou mais na USF e aproximadamente $60 \%$ trabalhavam 33 horas ou mais no serviço. Observou-se que a categoria profissional com maior frequência foram os técnicos de enfermagem $(35,9 \%)$, seguida dos agentes comunitários de saúde (28,8\%). Os enfermeiros representaram $11,5 \%(n=59)$ do total; destes, $23,7 \%$ referiram serem coordenadores da unidade (Tabela 1). Tabela 1.

\begin{tabular}{|c|c|c|c|}
\hline Categoria profissional & $\mathbf{n}$ & Total de questionários preenchidos & Taxa de resposta (\%) \\
\hline Enfermeiro & 97 & 59 & 60,8 \\
\hline Agente comunitário de saúde & 251 & 148 & 58,9 \\
\hline Cirurgião-dentista & 65 & 31 & 50,7 \\
\hline Técnico de enfermagem & 414 & 184 & 44,7 \\
\hline Técnico administrativo & 69 & 29 & 44,9 \\
\hline Auxiliar de odontologia & 116 & 34 & 29,3 \\
\hline Médico & 119 & 28 & 23,5 \\
\hline
\end{tabular}

Fonte: Os autores.

$\mathrm{Na}$ avaliação geral em segurança do paciente, considerando os sistemas e processos clínicos que o serviço utiliza para prevenir, identificar e corrigir problemas, percebeu-se que estes foram avaliados como muito bom ou excelente por $35,5 \%$ dos profissionais. Ademais, a melhor avaliação (muito bom ou 
excelente) foi realizada pelos profissionais da odontologia e enfermeiros, e a pior avaliação (ruim ou razoável) pelos técnicos de enfermagem e agentes comunitários de saúde (Tabela 2).

Destaca-se que a análise de confiabilidade do questionário, utilizando-se do alfa de Cronbach, demonstrou valores superiores a 0,65 para todas as seções, com um alfa de Cronbach total (incluindo todas as seções do instrumento) de 0,887. Tabela 2.

\begin{tabular}{|c|c|c|c|}
\hline \multirow{3}{*}{ Categoria profissional* } & \multicolumn{3}{|c|}{ Avaliação geral (segurança do paciente) } \\
\hline & $\begin{array}{c}\text { Ruim } \\
\text { Razoável }\end{array}$ & Bom & $\begin{array}{c}\text { Muito bom/ } \\
\text { Excelente }\end{array}$ \\
\hline & $\mathrm{n}(\%)$ & $\mathrm{n}(\%)$ & $\mathrm{n}(\%)$ \\
\hline Técnico em enfermagem & $43(24,0)$ & $70(39,1)$ & $66(36,9)$ \\
\hline Agente comunitário de saúde & $31(21,1)$ & $75(51,0)$ & $41(27,9)$ \\
\hline Odontologia & $10(15,9)$ & $25(39,7)$ & $28(44,4)$ \\
\hline Enfermeiro & $6(10,5)$ & $23(40,4)$ & $28(49,1)$ \\
\hline Equipe administrativa & $3(10,3)$ & $19(65,5)$ & $7(24,1)$ \\
\hline Médico & $3(11,5)$ & $15(57,7)$ & $8(30,8)$ \\
\hline Total & $96(19,2)$ & $227(45,3)$ & $178(35,5)$ \\
\hline
\end{tabular}

$\frac{\text { Total }}{{ }^{*} \text { A avaliação geral foi respondida por } 493 \text { entrevistados. }}$

Fonte: Os autores.

Com base na análise de respostas positivas, neutras e negativas, destaca-se que os técnicos de enfermagem, agentes comunitários de saúde e equipe administrativa apresentaram cultura de segurança do paciente mais fragilizada nas dimensões comunicação e acompanhamento dos pacientes, processo de trabalho no serviço de saúde e avaliação global da qualidade do cuidado em relação às demais categorias profissionais. Além disso, os agentes comunitários de saúde, médicos, pessoal da equipe administrativa e técnicos de enfermagem apresentaram cultura de segurança mais fragilizada em relação aos demais profissionais na dimensão serviço de saúde de atuação. $O$ apoio de gestores apresentou cultura de segurança mais fragilizada pela equipe de odontologia e administrativa (Tabela 3).

Nas análises de associação, não se observou relação estatisticamente significativa entre o processo de trabalho no serviço de saúde com qualquer categoria profissional. Percebeu-se que profissionais técnicos de enfermagem avaliaram de forma mais fragilizada as dimensões comunicação e acompanhamento do paciente (RP: 1,21; IC95\%: 1,06-1,39) e serviço de saúde de atuação (RP: 1,28; IC95\%: 1,03-1,60) em comparação com os demais profissionais. De forma contrária, os profissionais de odontologia (RP: 0,58; IC95\%: 0,40-0,90) e os enfermeiros (RP: 0,60; IC95\%: 0,40-0,92) apresentaram avaliação mais fortalecida da dimensão serviço de saúde de atuação (Tabela 4).

Em relação à avaliação geral da qualidade do cuidado, enfermeiros (RP: 0,47; IC95\%: 0,25-0,86) apresentaram percepção mais fortalecida e equipe administrativa (RP: 1,62; IC95\%: 1,07-2,45) mais fragilizada que as demais categorias profissionais. 
A análise relativa ao apoio de gestores demonstrou que os profissionais do serviço de odontologia (RP: 1,11; IC95\%: 1,03-1,19) apresentaram um julgamento mais fragilizado que os outros profissionais (Tabela 4).

Tabela 3.

Distribuição da percepção sobre a cultura de segurança (fragilizada) do paciente segundo categoria profissional, atenção primária à saúde, Londrina, PR, 2017.

\begin{tabular}{|c|c|}
\hline Variável & Dimensão da cultura de segurança fragilizada $n(\%)$ \\
\hline \multicolumn{2}{|c|}{ Processo de trabalho no serviço de saúde } \\
\hline Técnico de enfermagem & $168(91,3)$ \\
\hline Agente comunitário de saúde & $138(93,2)$ \\
\hline Odontologia & $58(89,2)$ \\
\hline Enfermeiro & $51(76,4)$ \\
\hline Equipe administrativa & $27(93,1)$ \\
\hline Médico & $24(75,7)$ \\
\hline \multicolumn{2}{|c|}{ Comunicacação e acompanhamento do paciente } \\
\hline Técnico de enfermagem & $117(63,6)$ \\
\hline Agente comunitário de saúde & $88(59,5)$ \\
\hline Odontologia & $30(46,2)$ \\
\hline Enfermeiro & $25(42,4)$ \\
\hline Equipe administrativa & $18(62,1)$ \\
\hline Médico & $11(40,7)$ \\
\hline \multicolumn{2}{|l|}{ Serviço de saúde de atuação } \\
\hline Técnico de enfermagem & $82(50,9)$ \\
\hline Agente comunitário de saúde & $81(63,3)$ \\
\hline Odontologia & $18(28,6)$ \\
\hline Enfermeiro & $16(30,2)$ \\
\hline Equipe administrativa & $13(54,2)$ \\
\hline Médico & $17(60,7)$ \\
\hline \multicolumn{2}{|c|}{ Avaliação geral da qualidade do cuidado } \\
\hline Técnico de enfermagem & $58(32,4)$ \\
\hline Agente comunitário de saúde & $58(39,5)$ \\
\hline Odontologia & $14(21,9)$ \\
\hline Enfermeiro & $9(15,5)$ \\
\hline Equipe administrativa & $13(44,8)$ \\
\hline Médico & $5(19,2)$ \\
\hline \multicolumn{2}{|l|}{ Apoio dos gestores* } \\
\hline Técnico de enfermagem & $118(72,0)$ \\
\hline Agente comunitário de saúde & $96(71,1)$ \\
\hline Odontologia & $48(80,0)$ \\
\hline Enfermeiro & $23(69,7)$ \\
\hline Equipe administrativa & $23(88,5)$ \\
\hline Médico & $19(70,4)$ \\
\hline
\end{tabular}

Fonte: Os autores. 
Tabela 4.

Associação bruta e ajustada entre dimensões da cultura de segurança do paciente (fragilizada) e categoria profissional, atenção primária à saúde, Londrina, PR, 2017.

\begin{tabular}{|c|c|c|}
\hline \multirow{3}{*}{ Variável independente } & \multicolumn{2}{|c|}{ Dimensões da cultura de segurança fragilizadas } \\
\hline & Análise bruta & Análise ajustada \\
\hline & RP (IC95\%) & RP $($ IC95\%) \\
\hline \multicolumn{3}{|c|}{ Processo de trabalho no serviço de saúde } \\
\hline Técnico de enfermagem & $1,02(0,96-1,08)$ & $1,05(0,99-1,12)$ \\
\hline Agente comunitário de saúde & $1,05(0,99-1,10)$ & $1,02(0,95-1,09)$ \\
\hline Odontologia & $0,99(0,90-1,08)$ & $0,96(0,87-1,06)$ \\
\hline Enfermeiro & $0,93(0,83-1,04)$ & $0,94(0,84-1,05)$ \\
\hline Equipe administrativa & $1,03(0,93-1,14)$ & $1,04(0,93-1,16)$ \\
\hline Médico & $0,95(0,81-1,10)$ & $0,95(0,81-1,11)$ \\
\hline \multicolumn{3}{|c|}{ Comunicação e acompanhamento do paciente } \\
\hline Técnico de enfermagem & $1,18(1,04-1,34)^{* *}$ & $1,21(1,06-1,39)^{* *}$ \\
\hline Agente comunitário de saúde & $1,01(0,88-1,16)$ & $1,00(0,85-1,19)$ \\
\hline Odontologia & $0,88(0,71-1,09)$ & $0,88(0,70-1,11)$ \\
\hline Enfermeiro & $0,78(0,61-1,01)$ & $0,79(0,61-1,02)$ \\
\hline Equipe administrativa & $1,13(0,90-1,43)$ & $1,13(0,88-1,46)$ \\
\hline Médico & $0,69(0,45-1,05)$ & $0,70(0,49-1,05)$ \\
\hline \multicolumn{3}{|l|}{ Serviço de saúde de atuação } \\
\hline Técnico de enfermagem & $1,07(0,89-1,30)$ & $1,28(1,03-1,60)^{*}$ \\
\hline Agente comunitário de saúde & $1,39(1,16-1,67)^{* * *}$ & $1,21(0,98-1,50)$ \\
\hline Odontologia & $0,54(0,37-0,81)^{* *}$ & $0,58(0,40-0,90)^{*}$ \\
\hline Enfermeiro & $0,59(0,39-0,89)^{*}$ & $0,60(0,40-0,92)^{*}$ \\
\hline Equipe administrativa & $1,19(0,83-1,71)$ & $1,08(0,77-1,51)$ \\
\hline Médico & $1,20(0,87-1,66)$ & $1,07(0,78-1,48)$ \\
\hline \multicolumn{3}{|c|}{ Avaliação geral da qualidade do cuidado } \\
\hline Técnico de enfermagem & $1,11(0,85-1,45)$ & $1,27(0,95-1,71)$ \\
\hline Agente comunitário de saúde & $1,38(1,06-1,80)^{*}$ & $1,27(0,92-1,75)$ \\
\hline Odontologia & $0,67(0,42-1,09)$ & $0,75(0,45-1,24)$ \\
\hline Enfermeiro & $0,49(0,26-0,88)^{*}$ & $0,47(0,25-0,86)^{*}$ \\
\hline Equipe administrativa & $1,57(1,04-2,37)^{*}$ & $1,62(1,07-2,45)^{*}$ \\
\hline Médico & $0,62(0,28-1,37)$ & $0,59(0,16-1,31)$ \\
\hline \multicolumn{3}{|l|}{ Apoio dos gestores ${ }^{a}$} \\
\hline Técnico de enfermagem & $0,99(0,93-1,07)$ & $0,99(0,92-1,06)$ \\
\hline Agente comunitário de saúde & $0,94(0,86-1,01)$ & $0,93(0,84-1,03)$ \\
\hline Odontologia & $1,13(1,07-1,18)^{* * *}$ & $1,11(1,03-1,19)^{* *}$ \\
\hline Enfermeiro & $0,94(0,82-1,09)$ & $0,96(0,84-1,10)$ \\
\hline Equipe administrativa & $1,09(1,01-1,19)^{*}$ & $1,09(0,99-1,21)$ \\
\hline Médico & $1,00(0,86-1,15)$ & $1,01(0,87-1,17)$ \\
\hline
\end{tabular}




\section{Discussão}

A avaliação geral de segurança do paciente foi respondida de maneira mais negativa (ruim ou razoável) por técnicos de enfermagem eagentes comunitários de saúde, e de maneira mais positiva (muito bom ou excelente) por profissionais da odontologiaeenfermeiros.Entende-sequeostécnicosdeenfermagem eagentes comunitários sãotrabalhadores sobrecarregados na APS (Silva e Menezes, 2008; Suehiro et al., 2008), o que pode facilitar para uma avaliação e percepção mais fragilizada quanto à segurança do paciente (Astier-Peña et al., 2015).

Os técnicos de enfermagem foram os profissionais que mais avaliaram a comunicação e o acompanhamento do paciente e aspectos quanto ao serviço de atuação de forma mais fragilizada. Vale ressaltar que essa categoria profissional é, na APS, uma das principais atingidas com a sobrecarga de trabalho e esgotamento profissional devido ao constante e estreito contato com a população assistida (Silva e Menezes, 2008; Suehiro et al., 2008; Martins, 2011; Trindade, Lauter e Beck, 2009). Tendo em vista essa possível sobrecarga de trabalho e constante exposição a fatores estressores (Belancieri, 2004, 2007; Belancieri et al., 2010; Scherer et al., 2016), entende-se ser natural que tais profissionais tenham percepção mais fragilizada do seu serviço de saúde.

É importante destinar atenção à saúde física e emocional do trabalhador, pois, quando esgotado, ele tem autoestima prejudicada e deterioração do sentimento de competência, o que leva à diminuição de habilidades técnicas desses profissionais, além de ser fator contribuinte para absenteísmo e presenteísmo, o que prejudica a qualidade da assistência em saúde (Rodrigues, Santos e Souza, 2017; Telles e Pimenta, 2009).

Com relação ao serviço de saúde de atuação, os profissionais enfermeiros apresentaram menor prevalência de cultura de segurança fragilizada quando comparados às demais categorias profissionais. Percebeu-se ainda avaliação mais fortalecida dos enfermeiros quanto à avaliação geral da qualidade do cuidado. Estudo transversal realizado com enfermeiros de um hospital do município de Vitória (ES) também identificou que profissionais enfermeiros percebem a segurança do paciente como algo positivo: $89,0 \%$ deles acreditam que os erros cometidos são tratados de forma apropriada, apesar de reconhecerem que ainda existem pontos a serem melhorados (Matiello et al., 2016). Em estudo qualitativo realizado com enfermeiros da atenção hospitalar e da APS quanto à segurança do paciente e aos erros de medicação no estado de São Paulo, identificou-se que enfermeiros hospitalares entendem possuir atuação mais hierárquica, de autoridade, e que enfermeiros da APS se percebem como apoiadores e coordenadores do serviço (Dias et al., 2014). 
Nesse sentido, infere-se que enfermeiros da APS, por ocuparem função de coordenação do cuidado e por perceberem-se como apoiadores da equipe de enfermagem, entendem que são os profissionais mais responsáveis pela garantia da assistência segura no serviço de saúde. Além disso, eles conhecem as estratégias e práticas pensadas e aplicadas para solucionar os obstáculos identificados no processo de trabalho com relação ao tema. Assim, podem apresentar percepção mais fortalecida com relação à segurança do paciente.

Os profissionais administrativos apresentaram maior prevalência de percepção fragilizada da segurança do paciente do que os demais profissionais em relação à avaliação geral da qualidade do cuidado. Tal percepção pode ser decorrente da menor aproximação que profissionais da equipe administrativa têm com o processo de trabalho em saúde se comparados aos demais trabalhadores, o que os distancia da maioria dos processos e condições de trabalho do serviço, que são aqueles relacionados ao cuidado à saúde, levando-os a desconhecê-los e assim avaliá-los de forma fragilizada (Rodrigues, Santos e Souza, 2017; Carnelutti, 2012; Gonçalves, 2011). Pode ser ainda decorrente da frustração profissional quanto à área de atuação, uma vez que esses profissionais podem apresentar preferência por não atenderem diretamente os pacientes, mas desejariam atuar de forma a interagir somente com o ambiente e os equipamentos utilizados, numa área essencialmente burocrática.

Também se percebeu que profissionais de odontologia apresentaram menor prevalência de avaliação fragilizada da cultura de segurança em relação ao serviço de saúde de atuação. A recente inclusão dos cirurgiões-dentistas na Estratégia Saúde da Família (ano 2000) coopera para um vínculo pouco sólido com os demais profissionais e pacientes e o desconhecimento do processo de trabalho (Boaretto, 2011). Ademais, em geral, no município de Londrina, os serviços de odontologia localizados na APS têm dinâmica própria, sendo, na maioria das vezes, distanciados das outras atividades realizadas nas UBSs. Assim, com frágil inserção na equipe, os profissionais tendem a desconhecer o processo de trabalho e seus problemas quanto à segurança do paciente, realizando uma avaliação mais positiva desses aspectos. Essa explicação pode também ser um dos motivos da avaliação mais fragilizada dos profissionais dos serviços de odontologia em relação ao apoio dos gestores, pois suas atividades são consideradas destoantes em relação às que ocorrem na UBS como um todo, sem envolvimento direto dos demais profissionais e, em especial, dos coordenadores das unidades.

A prática da segurança do paciente na APS tem sido identificada como um desafio no contexto brasileiro. Em consonância com a identificação desse desafio, soluções têm sido propostas, tais quais a inserção e o envolvimento de pacientes e família no planejamento de estratégias para a promoção de segurança e na avaliação da segurança do paciente; a implementação de tecnologias de informação na rotina de assistência, assim como prontuários eletrônicos, boletins de 
acompanhamento, aumento do uso de tecnologias leves nas relações interpessoais e em educação profissional (Marchon, Mendes Júnior e Pavão, 2015), que podem cooperar para o atendimento humanizado, além de serem fortalecedoras da cultura de segurança do paciente (Brasil, 2006).

Identificou-se em uma pesquisa realizada no campus da Universidade Federal de São Paulo (Unifesp), a qual oferece cursos nas áreas de enfermagem, fisioterapia, medicina e farmácia, que os conteúdos relacionados à segurança do paciente são abordados de forma fragmentada, não se consolidando em aprendizado significativo dos graduandos. Acredita-se que, tal qual a Unifesp, a maioria das universidades não dispõe de tempo e outros recursos suficientes para tratar do tema segurança do paciente de forma satisfatória, já que este é um assunto recente na realidade brasileira (Bohomol, Freitas e Cunha, 2016). Entretanto, salienta-se que a abordagem desse tema nas graduações da área da saúde é de extrema importância.

Dentre as fragilidades do presente estudo, destaca-se a taxa de resposta, a qual foi inferior a 50,0\%, especialmente entre médico e auxiliares de odontologia. Porém, verifica-se que a maioria dos estudos com metodologia semelhante apresenta taxas compatíveis. Além disso, não foram avaliadas no presente estudo todas as dimensões da cultura de segurança do paciente propostas pelo instrumento MOSPSC. Como aspectos positivos, todavia, devem ser salientados a coleta de dados primários, envolvendo todo um município de grande porte do Brasil; a boa consistência interna aferida por meio do Alfa de Cronbach; e a abordagem de várias categorias profissionais, até então incomum na maioria dos estudos com essa temática.

Percebe-se, com base neste estudo, a real necessidade do refazer de práticas, percepções e quiçá valores profissionais prevalentes nos serviços de saúde de atenção primária do município, movimentando seu sistema simbólico e consequentemente proporcionando uma cultura de segurança do paciente mais qualificada nesse nível de atenção. Com o planejamento conjunto de estratégias de assistência, a responsabilidade de solucionar as situações de saúde não ficará direcionada somente a alguns profissionais, sobrecarregando-os, mas será dividida com toda a equipe, unindo diversos saberes para a solução desses casos. Assim, os membros da equipe se apoiarão mutuamente, fortalecendo seus vínculos interprofissionais para atingir o objetivo principal da assistência à saúde: oferecer cuidado qualificado e humanizado, além da promoção da saúde da população.

É importante ainda que, na rotina de trabalho da APS, os trabalhadores sejam corresponsáveis pelo diagnóstico de erros e planejamento de estratégias de combate a estes, para que se sintam parte do processo como um todo e não somente da implementação e prática de ações mais seguras. Com o estímulo dos trabalhadores à prática da segurança do paciente na atenção primária e seu consequente aperfeiçoamento, espera-se, além de produzir uma assistência segura 
à saúde, aumentar a confiabilidade e efetividade dos serviços e, ainda, que se intensifique o vínculo do usuário com os profissionais.

Para que haja o planejamento conjunto de estratégias de assistência que promovam a segurança do paciente, é necessário que o trabalho em equipe seja priorizado. Dessa forma, os profissionais que ainda não têm inclusão sólida na equipe de saúde, ou ainda que desconhecem o processo de trabalho, devem ser incluídos e integrados na equipe de forma completa (Peruzzo et al., 2018).

\section{Conclusões}

O estudo aqui apresentado verificou uma baixa avaliação positiva (muito bom ou excelente) da segurança do paciente. Além disso, o processo de trabalho no serviço de atuação e apoio dos gestores foram as dimensões da cultura de segurança com avaliação mais fragilizada. Percebeu-se ainda que os técnicos deenfermagemforamaquelesqueapresentaramavaliaçãomaisfragilizadaem relaçãoàcomunicaçãoeaoacompanhamentodopacienteeserviçodesaúdede atuação. Deforma contrária, os profissionais enfermeiros apresentaram avaliação mais fortalecida da segurança do paciente.

Sugere-se a realização de estudos mais frequentes com APS, para refutar ou não os achados da presente investigação, mas também que possam avaliar a prevalência de erros relacionados ao processo de cuidado nesse nível de atenção.

\section{LA CULTURA SOBRE LA SEGURIDAD DEL PACIENTE EN LA ATENCIÓN PRIMARIA DE SALUD: DISTINCIONES ENTRE CATEGORÍAS PROFESIONALES}

Resumen El estudio que originó este artículo tuvo como objetivo analizar la cultura de seguridad del paciente en la atención primaria de salud de un municipio de gran población en el estado de Paraná, Brasil, según la categoría profesional. Se desarrolló un estudio transversal con trabajadores de la atención primaria, con recolección de datos realizada en 2017, por medio de la aplicación de un instrumento autorrellenable denominado Pesquisa sobre Cultura de Seguridad del Paciente para la Atención Primaria (Pesquisa sobre Cultura de Segurança do Paciente para Atenção Primária, en portugués). Los resultados indicaron que el proceso de trabajo en el servicio de actuación y el apoyo de los gestores fueron las dimensiones de la cultura de seguridad con evaluación más fragilizada. De modo general, hubo divergencias en la percepción de la cultura de seguridad por los profesionales de salud de la atención primaria, denotando la importancia de la planificación conjunta de estrategias de asistencia a la salud. Los técnicos en enfermería presentaron mayor prevalencia de evaluación fragilizada de la cultura de seguridad del paciente. De modo contrario, los enfermeros presentaron menor prevalencia de evaluación fragilizada sobre la cultura de seguridad.

Palabras clave seguridad del paciente; atención primaria de salud; trabajadores de salud. 


\section{Colaboradores}

Lilian Louzada Macedo, Edmarlon Girotto e Ana Maria Rigo Silva idealizaram o estudo. Lilian Louzada Macedo e Edmarlon Girotto participaram igualmente de todas as fases de elaboração do manuscrito e aprovaram a versão final do artigo. Ana Maria Rigo Silva, Maria do Carmo Fernandez Lourenço Haddad e João Felipe Marques da Silva participaram da redação, revisão crítica e aprovação da versão final do artigo.

\section{Financiamento}

Não houve financiamento para a execução da pesquisa.

\section{Referências}

AGENCY FOR HEALTHCARE RESEARCH AND QUALITY (AHRQ). Medical Office Survey on Patient Safety Culture. jun. 2016. Disponível em: <http://www.ahrq.gov/professionals/ quality-patient-safety/patientsafetyculture/ medical-office/index.html>. Acesso em: 15 nov. 2016.

ASTIER-PEÑA, Maria P. et al. Are Spanish primary care professional aware of patient safety? European Journal of Public Health, Estocolmo, v. 5, n. 25, p. 781-787, abr. 2015.

BELANCIERI, Maria F. Estresse e repercussões psicossomáticas em trabalhadores da enfermagem de um hospital universitário. Texto \& Contexto Enfermagem, Florianópolis, v. 13, n. 1, p. 124-131, jan.-mar. 2004.

BELANCIERI, Maria F. Promoção do processo de resiliência em enfermeiras: uma possibilidade. 203f. Tese (Doutorado em Psicologia) - Pontifícia Universidade Católica de São Paulo, São Paulo, 2007.

BELANCIERI, Maria F. et al. A resiliência em trabalhadores da área da enfermagem. Estudos de Psicologia, Campinas, v. 2, n. 27, p. 227233, abr.-jun. 2010.
BOARETTO, Patrícia P. A inclusão da odontologia na Estratégia Saúde da Família. 32f. Trabalho de Conclusão de Curso (Especialização em Atenção Básica) - Universidade Federal de Minas Gerais, Belo Horizonte, 2011.

BOHOMOL, Elena; FREITAS, Maria A. O.; CUNHA, Isabel C. K. O. Ensino da segurança do paciente na graduação em saúde: reflexões sobre saberes e fazeres. Interface: Comunicação, Saúde, Educação, Botucatu, v. 20, n. 58, p. 727-741, mar. 2016.

BRASIL. Ministério da Saúde. Secretaria de Atenção à Saúde. Núcleo Técnico da Política Nacional de Humanização. HumanizaSUS: documento base para gestores e trabalhadores do SUS. 3. ed. Brasília: Ministério da Saúde, 2006.

BRASIL. Ministério da Saúde. Departamento de Atenção Básica. Nos últimos 4 anos, saúde destinou $R \$ 5$ bilhões para reforma e ampliação de 26 mil postos de saúde. 2015. Disponível em: <http://www.blog.saude.gov.br/index. $\mathrm{php} /$ geral/35215-nos-ultimos-4-anos-saudedestinou-r-5-bilhoes-para-reforma-e-ampliacaode-26-mil-postos-de-saude $>$. Acesso em: $1{ }^{\circ}$ nov. 2018. 
BRASIL. Portaria n. 2.436, de 21 de setembro de 2017. Aprova a Política Nacional de Atenção Básica. Diário Oficial da União, Brasília, DF, 22 set. 2017, seção 1, p. 68.

CARNELUTTI, Caroline. As fragilidades profissionais do enfermeiro gestor da UBS. $17 f$. Monografia (Especialização em Gestão de Organização Pública em Saúde) - Universidade Federal de Santa Maria, Santa Maria, 2012.

CRONBACH, Lee J. Coefficient alpha and the internal structure of tests. Psychometrika, Colorado Springs, v. 16, n. 3, p. 297-334, set. 1951.

DIAS, Jéssica D. et al. Compreensão de enfermeiros sobre segurança do paciente e erros de medicação. REME: Revista Mineira de Enfermagem, Belo Horizonte, v. 18, n .4, p. 866-873, out.-dez. 2014.

GEHRING, Katrin et al. Frequency of and harm associated with primary care safety incidents. American Journal of Managed Care, Old Brigde, v. 18, n. 9, p. 323-337, set. 2012.

GONÇALVES, Sirlane M. S. Papel do enfermeiro na unidade básica de saúde: assistência a saúde ou gerência de ações? 40f. Monografia (Especialização em Atenção Básica em Saúde da Família), Universidade Federal de Minas Gerais, Belo Horizonte, 2011.

HUBERT, Patricia M. Application of Jean Watson's Theory of Transpersonal Caring in nurses practicing in a pain center. 100f. Thesis (Doctorate of Nursing Practice) - Seton Hall University, New Jersey, 2018.

INSTITUTO BRASILEIRO DE GEOGRAFIA E ESTATÍSTICA (IBGE). População. Disponível em: $<$ https://cidades.ibge.gov.br/brasil/pr/ londrina/panorama>. Acesso em: 18 dez. 2018.

KLEIN, Carlos H.; BLOCH, Katia V. Estudos seccionais. In: MEDRONHO, Roberto A. et al. (orgs.). Epidemiologia. 2. ed. São Paulo: Atheneu, 2008. p. 195-200.
LUIZ, Olinda C.; COHN, Amélia. Sociedade de risco e risco epidemiológico. Cadernos de Saúde Pública, Rio de Janeiro, v. 22, n. 11, p. 2.339-2.348, nov. 2006.

MARCHON, Simone G. A segurança do paciente na atenção primária à saúde. 78f. Tese (Doutorado em Saúde Pública) - Escola Nacional de Saúde Pública Sergio Arouca, Fundação Oswaldo Cruz, Rio de Janeiro, 2014.

MARCHON, Simone G.; MENDES JÚNIOR, Walter V.; PAVÃO, Ana L. B. Características dos eventos adversos na atenção primária à saúde no Brasil. Cadernos de Saúde Pública, Rio de Janeiro, v. 31, n. 11, p. 2.313-2.330, nov. 2015.

MARTINS, Leonardo F. Estresse ocupacional e esgotamento profissional entre profissionais da atenção primária à saúde. 140f. Dissertação (Mestrado em Psicologia) - Instituto de Ciências Humanas, Universidade Federal de Juiz de Fora, Juiz de Fora, 2011.

MATIELLO, Raquel D. C. et al. A cultura de segurança do paciente na perspectiva do enfermeiro. Cogitare Enfermagem, Curitiba, v. 21, n. 5, p.1-9, fev.-jun. 2016.

PERUZZO, Hellen E. et al. Os desafios de se trabaIhar em equipe na Estratégia Saúde da Família. Escola Anna Nery Revista de Enfermagem, Rio de Janeiro, v. 22, n. 4, p. 1-9, jun. 2018.

PAESE, Fernanda; DAL SASSO, Grace T. M. Cultura da segurança do paciente na atenção primária à saúde. Texto \& Contexto Enfermagem, Florianópolis, v. 2, n. 22, p. 302-310, abr-jun. 2013.

RODRIGUES, Cláudia C. F. M.; SANTOS, Viviane E. P.; SOUZA, Paulo. Segurança do paciente e enfermagem: interface com estresse e síndrome de burnout. Revista Brasileira de Enfermagem, Brasília, v. 70, n. 5, p. 1.1411.147, set.-out. 2017. 
SCHERER, Magda D. A. et al. Aumento das cargas de trabalho em técnicos de enfermagem na atenção primária à saúde no Brasil. Trabalho, Educação e Saúde, Rio de Janeiro, v. 14, n. 1, p. 89-104, nov. 2016.

SILVA, Andréa T. C; MENEZES, Paulo R. Esgotamento profissional e transtornos mentais comuns em agentes comunitários de saúde. Revista de Saúde Pública, São Paulo, v. 42, n. 45, p. 921-929, out. 2008.

SORRA, Joann; FRANKLIN, Martha; STREAGLE, Suzanne. Medical Office Survey on Patient Safety Culture. Rockville: Agency for Healthcare Research and Quality, 2008. Disponível em: $<$ https://sopsdatabase.ahrq.gov/DB/Public/ Documents/mosurveyguide.pdf $>$. Acesso em: 16 ago. 2019.

SUEHIRO, Adriana C. B. et al. Vulnerabilidade ao estresse e satisfação no trabalho em profissionais do Programa de Saúde da Família. Boletim de Psicologia, São Paulo, v. 58, n. 129, p. 206-218, dez. 2008.
TELLES, Heloísa; PIMENTA, Ana M. C. Síndrome de burnout em agentes comunitários de saúde e estratégias de enfrentamento. Saúde e Sociedade, São Paulo, v. 18, n. 3, p. 467478, set. 2009.

TIMM, Márcia. Adaptação transcultural do instrumento Medical Office Survey on Patient Safety Culture sobre segurança do paciente para atenção primária à saúde. 146f. Dissertação (Mestrado em Enfermagem) - Faculdade de Ciências da Saúde, Universidade de Brasília, Brasília, 2015.

TIMM, Márcia; RODRIGUES, Maria C. S. Adaptação transcultural de instrumento de cultura de segurança para a atenção primária. Acta Paulista de Enfermagem, São Paulo, v. 29, n. 1, p. 26-37, jan. 2016.

TRINDADE, Letícia L.; LAUTERT, Liana; BECK, Carmem L. C. Mecanismos de enfrentamento utilizados por trabalhadores esgotados e não esgotados da Estratégia de Saúde da Família. Revista Latino-Americana de Enfermagem, Ribeirão Preto, v. 17, n. 5, p. 1-9, set.-out. 2009. 